\title{
Pacific
}

Journal of

Mathematics

\section{$q$-CANONICAL COMMUTATION RELATIONS AND STABILITY OF THE CUNTZ ALGEBRA}

Palle E. T. Jorgensen, L. M. Schmitt And Reinhard Frank Werner 


\title{
q-CANONICAL COMMUTATION RELATIONS AND STABILITY OF THE CUNTZ ALGEBRA
}

\author{
P. E. T. JøRgensen, L. M. Schmitt AND R. F. Werner
}

We consider the $q$-deformed canonical commutation relations $a_{i} a_{j}^{*}$ - $q a_{j}^{*} a_{i}=\delta_{i j} \mathbb{1}, i, j=1, \ldots, d$, where $d$ is an integer, and $-1<q<1$. We show the existence of a universal solution of these relations, realized in a $C^{*}$-algebra $\mathscr{E}^{q}$ with the property that every other realization of the relations by bounded operators is a homomorphic image of the universal one. For $q=0$ this algebra is the Cuntz algebra extended by an ideal isomorphic to the compact operators, also known as the Cuntz-Toeplitz algebra. We show that for a general class of commutation relations of the form $a_{i} a_{j}^{*}=\Gamma_{i j}\left(a_{1}, \ldots, a_{d}\right)$ with $\Gamma$ an invertible matrix the algebra of the universal solution exists and is equal to the Cuntz-Toeplitz algebra. For the particular case of the $q$-canonical commutation relations this result applies for $|q|<\sqrt{2}-1$. Hence for these values $\mathscr{E}^{q}$ is isomorphic to $\mathscr{E}^{0}$. The example $a_{i} a_{j}^{*}-q a_{i}^{*} a_{j}=\delta_{i j} \mathbb{1}$ is also treated in detail.

1. Introduction. In this paper we study the relations

$$
a_{i} a_{j}^{*}-q a_{j}^{*} a_{i}=\delta_{i j} \mathbb{1}, \quad i, j=1, \ldots, d,
$$

for bounded operators $a_{i}, i=1, \ldots, d, d<\infty$ on a Hilbert space, and a deformation parameter $q$ satisfying $-1<q<1$. For $q=0$ these relations are known as the Cuntz relations, and it is well known that in this case the $C^{*}$-algebra generated by the $a_{i}$ is essentially unique: it is either the so-called Cuntz-Toeplitz algebra, or the quotient of this algebra by its only closed two-sided ideal (generated by $\left.\mathbb{1}-\sum_{i} a_{i}^{*} a_{i}\right)$, which is known as the Cuntz algebra $\mathscr{O}_{d}$. Our main result in this paper is that the same statement holds for the relations (1) for small $q$. The technique we use also applies to more general relations of the form

$$
a_{i} a_{j}^{*}=\Gamma_{i j}\left(a_{1}, \ldots, a_{d}\right),
$$

where $\Gamma$ is an invertible matrix of functions in the functional calculus of $d$ variables, which satisfy a continuity and a growth condition specified below. We then show that one can decompose the generators as $a_{i}=v_{i} \rho$, where the $v_{i}$ satisfy the Cuntz relations, and $\rho$ satisfies 
an equation which can be solved by iteration. This iteration can be performed within the algebra generated by the $v_{i}$ implying that $\rho$ and $a_{i}$ are in the $C^{*}$-algebra generated by the $v_{i}$, and hence generate a Cuntz-Toeplitz or a Cuntz algebra.

In the recent physics literature there has been much interest in deformed versions of the canonical commutation relations for Bosons, partly as a tool for studying representations of the quantum groups [Bie, Mcf], but also as a possible generalization of the standard quantum description of indistinguishable particles [Gre]. The " $q$-relations" (1), or "quon $q$-mutation relations" [Gre], are of special interest because they interpolate between the canonical commutation relations for Bosons for $q=1$ (which do not have any bounded realizations), and the canonical anti-commutation relations for Fermions for $q=$ -1 . In these cases the relations are usually supplemented by the relations $a_{i} a_{j}-q a_{j} a_{i}=0$. However, for $|q|<1$ such additional relations led to an overdetermined system.

In the case of the Bose or Fermi relations, uniqueness questions have a long history. In the Bose case, where one usually writes the relations in terms of the exponentials of the $a_{i} \pm a_{i}^{*}$ (Weyl operators), a theorem of von Neumann [vNe] asserts uniqueness up to unitary equivalence for $d<\infty$. For infinitely many generators, which is the case of interest in quantum field theory, unitary equivalence breaks down. However, in every representation the $C^{*}$-algebra generated by the exponentials is $C^{*}$-isomorphic to the so-called CCR algebra, and is thus independent of the representation. Analogous results hold for the Fermionic case.

We are looking for a similar characterization of the representations of the $q$-relations up to $C^{*}$-isomorphism, where two representations are called $C^{*}$-isomorphic, if they generate isomorphic $C^{*}$-algebras, and the $C^{*}$-isomorphism between the two algebras can be chosen to map corresponding generators into each other. The result is expressed concisely in terms of a $C^{*}$-algebra $\mathscr{E}^{q}(d)$ with the universal property that any realization of the $q$-relations by bounded operators generates a homomorphic image of $\mathscr{E} q(d)$ (Proposition 3). Then the $C^{*}$-isomorphism classes of representations of the $q$-relations are in one-to-one correspondence to the closed two-sided ideals of $\mathscr{E}^{q}(d)$.

There is a special representation of the relations characterized by the property that it contains a cyclic vector $\Omega$ with $a_{i} \Omega=0$ for all $i$. Since each polynomial in the generators can be brought into a normal ("Wick ordered") form with all starred operators to the left 
of all unstarred ones by applying the $q$-relations, it is easy to see that the equation $a_{i} \Omega=0$ determines this representation uniquely. In analogy with ordinary Bose or Fermi commutation rules it will be called the Fock representation of the $q$-relations. In order to show that this is indeed a representation in a Hilbert space with positive definite scalar product, one has to show that $a_{i} \Omega=0$ implies the positivity of $\left\langle\Omega, X^{*} X \Omega\right\rangle$ for all polynomials $X$ in the variables $a_{i}$ and $a_{j}^{*}$. This rather non-trivial result has been obtained in [BS, Fiv].

Two cases of the $q$-relations can be studied in complete detail. The first is the case of a single relation $(d=1)$. Most of the physics literature treats either this case, or else commuting operators, each of which satisfies the $q$-relations separately. It is easy to see that, for $d=1$, the Fock representation is faithful, and contains only one proper two-sided ideal, which is generated by the one-dimensional projection onto the vacuum vector $\Omega$, and is isomorphic to the compact operators. The algebras $\mathscr{E}^{q}(1)$ are isomorphic, for all $q$, to the universal $C^{*}$-algebra $\mathscr{E}^{0}(1)$ generated by an isometry. $\mathscr{E}^{0}(1)$ is the Toeplitz algebra [Dou, Co1, Co2]. It is an extension [BDF] of $\mathscr{C}\left(S^{1}\right)$ by the algebra $\mathscr{K}$ of compact operators, i.e. there is an exact sequence $0 \rightarrow \mathscr{K} \rightarrow \mathscr{E}^{0}(1) \rightarrow \mathscr{C}\left(S^{1}\right) \rightarrow 0$. Under the isomorphisms $\mathscr{E}^{0}(1) \cong \mathscr{E}^{q}(1)$ the generators for different $q$ are related by multiplication with a suitable function of the number operator in Fock space, i.e. they are "weighted shifts" [Con]. It has been noted by several authors [Das, OKK] that such results are not specific to the $q$-relations, but also hold for more general relations like $a a^{*}=\Gamma\left(a^{*} a\right)$ for suitable functions $\Gamma$.

The second case which has been fully analyzed is the case $q=$ $0, d<\infty$. Each $a_{i}^{*}$ is then isometric, and the relations are essentially those stuided by [Cun]. Again we have that the Fock representation is faithful, and contains a single closed two-sided ideal, which is generated by $\mathbb{1}-\sum_{i} a_{i}^{*} a_{i}$, and is isomorphic to the compact operators $\mathscr{K}$. The quotient of $\mathscr{E}^{0}(d)$ by this ideal is a simple $C^{*}$-algebra known as the Cuntz algebra, and is usually denoted by $\mathscr{O}_{d}$. Again $0 \rightarrow \mathscr{K} \rightarrow \mathscr{E}^{0}(d) \rightarrow \mathscr{O}_{d} \rightarrow 0$ is exact. By analogy with the case $d=1$, we will call $\mathscr{E}^{0}(d)$ the Cuntz-Toeplitz algebra.

Combining these two well-known cases one is led for general $q \in$ $(-1,1)$ to the questions $(1)$ whether $\mathscr{E} q(d)$ is an extension of a simple $C^{*}$-algebra by the compact operators; $(2)$ whether the Fock representation of $\mathscr{E}^{q}(d)$ is faithful; (3) whether $\mathscr{E}^{q}(d)$ and $\mathscr{E}^{0}(d)$ are isomorphic for all $q$; and (4) whether such an isomorphism can be obtained 
by multiplying the generators of $\mathscr{E}^{0}(d)$ with a fixed $q$-dependent element of $\mathscr{E}^{0}(d)$.

Questions (3) and (4) make sense also for the more general " $\Gamma$ relations" of the form given in equation (2). For a universal $C^{*}$ algebra analogous to $\mathscr{E} q(d)$ to exist, the matrix of functions $\Gamma_{i j}$ has to satisfy an upper bound guaranteeing a uniform upper bound on any solution of equation (2). Assuming, in addition, a Lipschitz continuity estimate for $\Gamma_{i j}$, and, most importantly, a lower bound making the matrix $\Gamma\left(a_{1}, \ldots, a_{d}\right)$ invertible for all admissible arguments, we show in Theorem 9 that the universal solution of the $\Gamma$-relations (2) is of the form $a_{i}=v_{i} \rho$, where the $v_{i}$ are the generators in $\mathscr{E}^{\circ}(d)$, and $\rho \in \mathscr{E}^{0}(d)$ is a positive element computed from $\Gamma$. Hence (3) and (4) hold true, and the Cuntz-Toeplitz algebra $\mathscr{E}^{0}(d)$ is the universal $C^{*}$-algebra for all relations covered by our theorem.

We emphasize that our stability result does not fall within the range of analytic deformation theory (see [Ger], and Gerstenhaber's article in [HG], and references cited there), since the $\Gamma_{i j}$ need not be analytic functions. It is also not a case of isomorphisms of "close $C^{*}$-algebras" in the sense of [Chr], since we make explicit use of the fact that the "peturbed" algebra is given in terms of generators and relations of a specific type.

For the special case of the $q$-relations, the bounds needed in Theorem 9 are satisfied for $|q|<\sqrt{2}-1$. Thus the above conjectures (1) to (4) are valid for these values of $q$. Moreover, all information that has been accumulated about the Cuntz algebra [Eva, DR] becomes imediately relevant to the representations of the $q$-relations.

Our paper is organized as follows: In $\S 1$ we construct the universal algebra $\mathscr{E}^{q}(d)$ for the $q$-relations, and state some of its elementary properties. In $\S 2$ we prove the stability result for the Cuntz-Toeplitz algebra. In $\S 3$ we give some applciations of this result. Apart from the $q$-relations, we study in some detail the relations $a_{i} a_{j}^{*}-q a_{i}^{*} a_{j}=\delta_{i j} \mathbb{1}$ which differ form the $q$-relations in the position of the indices in the term containing $q$. For these modified relations we also compute the spectrum of $\sum_{i} a_{i}^{*} a_{i}$ in all possible bounded representations, and show that representations fail to exist for some negative values of $q$.

2. The $C^{*}$-algebra $\mathscr{E} q(\mathscr{H})$. In this section, we use a slightly different version of the $q$-relations, which makes the basic symmetries of these relations more transparent. Let $\mathscr{H}$ be a vector space with sesquilinear form $\langle\cdot, \cdot\rangle$, and let $q$ be a number with $-1<q<1$. We then study $\mathbb{C}$-linear maps $a: \mathscr{H} \rightarrow \mathscr{A}$ into a $C^{*}$-algebra $\mathscr{A}$ with 
identity such that

$$
a(f) a(g)^{*}-q a(g)^{*} a(f)=\langle g, f\rangle \mathbb{1}, \quad g, f \in \mathscr{H} .
$$

Any map $a$ with these properties will be called a representation, or a realization of the $q$-relations. The connection with the relations described in the introduction is then, that for some basis $\left\{e_{i}\right\} \subset \mathscr{H}$ which is orthonormal with respect to $\langle\cdot, \cdot\rangle$, we can write $a_{i}=a\left(e_{i}\right)$ and $a\left(\sum_{i} f_{i} e_{i}\right)=\sum_{i} f_{i} a_{i}$.

First, we need a result about the case of a single generator $a=a_{1}$ where $\mathscr{H}$ is one-dimensional. Further details about this case will be given below in Example 2.

Proposition 1. Let $\mathscr{A}$ be a $C^{*}$-algebra with unit, $q \in(-1,1)$, $c \in \mathbb{R}$, and $a \in \mathscr{A}$ a non-zero element satisfying

$$
a a^{*}-q a^{*} a=c \mathbb{1} \text {. }
$$

Then $c>0$, and either $a a^{*}=a^{*} a=c /(1-q) \mathbb{1}$, or the spectra of a $a^{*}$ and $a^{*} a$ are equal to the closure of the sequence

$$
c \frac{1-q^{n}}{1-q}
$$

where $n \in \mathbb{N}$, and $n \geq 0$ for $a^{*} a$ and $n \geq 1$ for $a a^{*}$.

Proof. We may assume $q \neq 0$. Let $\sigma(x)$ denote the spectrum of an element $x \in \mathscr{A}$. Then $\sigma\left(a a^{*}\right)=c+q \sigma\left(a^{*} a\right)$, and $\sigma\left(a a^{*}\right) \backslash\{0\}=$ $\sigma\left(a^{*} a\right) \backslash\{0\}$. Hence with $f(x)=c+q x$ we have for $x \neq 0$ that $x \in$ $\sigma\left(a a^{*}\right)$ if and only if $f(x) \in \sigma\left(a a^{*}\right)$. The sequence $f^{-n}(x), n \geq 0$, is unbounded unless $x=x_{\infty}=c(1-q)^{-1}$. Thus if $x_{\infty} \neq x \in \sigma\left(a a^{*}\right)$, the backwards iteration from $x$ must terminate in 0 . It follows that $x=f^{n}(0)$ for some $n \geq 1$. From the explicit solution of the forward iteration given in the proposition, one sees that the forward iterates are all non-zero; hence the entire sequence must be in $\sigma\left(a a^{*}\right)$. The exceptional vlaue $x_{\infty}$ is in the closure of the sequence. Since $a a^{*} \geq 0$ we get $c=(1-q) x_{\infty} \geq 0$.

It remains to exclude the possibility $c=0$. Since $a$ was assumed to be non-zero there must be some non-zero $x \in \sigma\left(a a^{*}\right)$. Hence with $c=0$ the whole sequence $q^{-n} x$ would be in $\sigma\left(a a^{*}\right)$. This contradicts the boundedness of $a$.

Corollary 2. Suppose $a: \mathscr{H} \rightarrow \mathscr{A}$ satisfies the q-relations. Then the sesquilinear form $\langle\cdot, \cdot\rangle$ on $\mathscr{H}$ is positive semidefinite, and 


$$
\begin{aligned}
\|a(f)\| & =c(q)\langle f, f\rangle^{1 / 2}, \quad \text { where } \\
c(q) & = \begin{cases}\frac{1}{\sqrt{1-q}} & \text { for } 0 \leq q<1, \\
1 & \text { for }-1<q \leq 0 .\end{cases}
\end{aligned}
$$

It follows that the map $a$ is continuous for the topology on $\mathscr{H}$ given by the seminorm $\|f\|=\langle f, f\rangle^{1 / 2}$ and the norm topology on $\mathscr{A}$. Therefore it extends by continuity to the completion of $\mathscr{H}$, and this extension again satisfies the $q$-relations. Hence there is no loss of generality in assuming that $\mathscr{H}$ is a Hilbert space, and we will make this assumption from now on.

An important consequence of Corollary 2 is that the norm bound on $a(f)$ is given by a constant independent of the particular realization of $a$. Only this fact is needed to construct the universal $C^{*}$-algebra associated with the $q$-relations, which is given in the following proposition.

Proposition 3. (1) For a given Hilbert space $\mathscr{H}$, and any $q \in$ $(-1,1)$, there exists a $C^{*}$-algebra, denoted by $\mathscr{E} q(\mathscr{H})$ with a map $a: \mathscr{H} \rightarrow \mathscr{E} q(\mathscr{H})$ satisfying the q-relations with the following universal

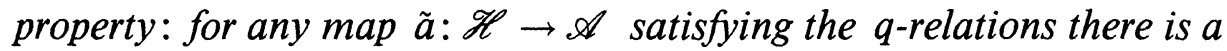
unique *-homomorphism $\tilde{\pi}: \mathscr{E} q(\mathscr{H}) \rightarrow \mathscr{A}$ such that $\tilde{\pi}(a(f))=\tilde{a}(f)$ for all $f \in \mathscr{H}$.

(2) $\mathscr{E} q(\mathscr{H})$ is determined up to $C^{*}$-isomorphism. For any isometry $V: \mathscr{H}_{1} \rightarrow \mathscr{H}_{2}$ between Hilbert spaces, there is a unique *-homomorphism $\mathscr{E} q(V): \mathscr{E} q\left(\mathscr{H}_{1}\right) \rightarrow \mathscr{E} q\left(\mathscr{H}_{2}\right)$ such that $\mathscr{E} q(V)(a(f))=a(V f)$. Thus $\mathscr{E} q$ is a covariant functor from the category of Hilbert spaces with isometries into the category of $C^{*}$-algebras with *-homomorphisms.

(3) $V \mapsto \mathscr{E}^{q}(V)$ is continuous on the unitary group of $\mathscr{H}$ in the sense that strong convergence $V_{\alpha} \rightarrow V$ implies $\mathscr{E}^{q}\left(V_{\alpha}\right)(X) \rightarrow$ $\mathscr{E} q(V)(X)$ in norm for all $X \in \mathscr{E}^{q}(\mathscr{H})$.

(4) $\mathscr{E} q(\mathscr{H})$ carries a natural $\mathbb{Z}$-grading, given by the family of closed subspaces

$$
\mathscr{E}^{q}(\mathscr{H})_{m}=\left\{X \in \mathscr{E}^{q}(\mathscr{H}) \mid \mathscr{E}^{q}(\eta \mathbb{1})(X)=\eta^{m} X \text { for } \eta \in \mathbb{C},|\eta|=1\right\}
$$

for $m \in \mathbb{Z}$. If $X_{m} \in \mathscr{E}^{q}(\mathscr{H})_{m}$ and $X_{n} \in \mathscr{E}^{q}(\mathscr{H})_{n}$, we have $X_{m} X_{n} \in$ $\mathscr{E} q(\mathscr{H})_{m+n}$, and the linear span of these subspaces is a dense subalgebra of $\mathscr{E} q(\mathscr{H})$. Explicitly, the projection onto $\mathscr{E} q(\mathscr{H})_{m}$ is given by

$$
\mathbb{P}_{m}(X)=\int d \eta \eta^{-m \mathscr{E} q}(\eta \mathbb{1})(X)
$$


where $d \eta$ denotes the normalized Haar measure on the circle.

Proof. (1), (2) Let $\mathbf{E}^{q}(\mathscr{H})$ denote the quotient of the $*$-algebra of non-commuting polynomials in the generators $a(f)$ by the ideal generated by the $q$-relations and the relations arising from the linearity of $a$. It is straightforward to check that $\mathbf{E}^{q}(\mathscr{H})$ satisfies the analogues of (1) and (2) in the category of $*$-algebras: if $\tilde{a}$ satisfies the relations, the homomorphism $\tilde{\pi}$ is defined simply by substituting the given $\tilde{a}(f)$ into any polynomial in $\mathbf{E}^{q}(\mathscr{H})$. In order to get a universal $\mathbf{C}^{*}$ algebra with the analogous property we have to define a $C^{*}$-seminorm $\|\cdot\|$ on $\mathbf{E}^{q}(\mathscr{H})$ with the property that for any bounded representation of the relations the substitution homomorphism is continuous, and hence extends to the completion $\mathscr{E} q(\mathscr{H})$ of $\mathbf{E}^{q}(\mathscr{H})$ with repect to that seminorm. This is to say that for any polynomial $X \in \mathbf{E}^{q}(\mathscr{H})$, and any bounded realization $\tilde{a}$ we must have $\|\tilde{\pi}(X)\| \leq\|X\|$. Hence we want to define

$$
\|X\|=\sup _{\tilde{a}}\|\tilde{\pi}(X)\|,
$$

where the sup is over all bounded realizations $\tilde{a}$ of the relations, and $\tilde{\pi}$ denotes the associated substitution homomorphism. There are three problems with this definition. The first is the technical point that the bounded realizations of the relations do not form a set, but a proper class. However, since $\|\tilde{\pi}(X)\|$ may be computed in the $C^{*}$-algebra generated by the $\tilde{a}(f)$, we may restrict the supremum to realizations in $C^{*}$-algebras with at most a certain cardinal number of elements depending on the number of generators via the dimension of $\mathscr{H}$. We now pick a Hilbert space of sufficiently high dimension such that all the universal representations of all these algebras can be realized in it. It is then clear that the supremum can be restricted to the set of realizations of the $q$-relations in bounded operators on this big Hilbert space.

The second problem is whether the supremum is possibly over the empty set, i.e. whether any bounded realizations of the relations exist at all. For the case at hand this question has been settled by [BS], who explicitly construct the so-called Fock representation for all $q \in$ $(-1,1)$. (See Example 1 below.)

The third problem is that the supremum might turn out to be infinite for some $X$. Since the $X \in \mathbf{E}^{q}(\mathscr{H})$ are polynomials in $\tilde{a}(f)$ this can be ruled out by proving an upper bound on $\|\tilde{a}(f)\|$, which is uniform with respect to all realizations $\tilde{a}$ of the $q$-relations. For the case at hand a bound of this kind was established in Corollary 2. Hence 
$\mathscr{E} q$ exists and has the universal property (1). The uniqueness and the further properties stated in (2) follow as usual from the universal property.

(3) It is clear from Corollary 2 that $V_{\alpha} \rightarrow V$ strongly, implies

$$
\mathscr{E}^{q}\left(V_{\alpha}\right)(a(f)) \rightarrow \mathscr{E}^{q}(V)(a(f))
$$

for all $f \in \mathscr{H}$. Hence we also have convergence for all polynomials $X$ in the variables $a(f), a(g)^{*}$, and by a straightforward estimate also the convergence for $X$ in the norm closure $\mathscr{E}^{q}(\mathscr{H})$ of the polynomials.

(4) The integral for $\mathbb{P}_{m}$ is well-defined and in $\mathscr{E} q(\mathscr{H})$ because by (3) the integrand is continuous in norm. On monomials in $a(f)$ and $a(g)^{*}$ we can compute the degree $m$ by counting the number of factors $a(f)$ and $a(f)^{*}$. Hence on monomials the additivity of the degree and the formula for $\mathbb{P}_{m}$ are obvious, and carry over to the completions $\mathscr{E} q(\mathscr{H})_{m}$ by continuity.

By (2), $\mathscr{E} q(\mathscr{H})$ depends only on the dimension of $\mathscr{H}$. Since we will later be mostly interested in the case $\operatorname{dim} \mathscr{H}<\infty$, we will write $\mathscr{E} q(d)$ for $\mathscr{E} q\left(\mathbb{C}^{d}\right)$.

EXAMPLE 1 (The Fock representation). Consider a representation of the $q$-relations by bounded operators in a Hilbert space $\mathscr{K}$. Let

$$
\mathscr{N}=\left\{\xi \in \mathscr{K} \mid \forall_{f \in \mathscr{H}} a(f) \xi=0\right\} .
$$

We call $\mathscr{N}$ the set of Fock vectors in this representation. Note that by applying the $q$-relations we can arrange any polynomial $X$ in the generators "in standard form", i.e. such that all $a(f)$ are to the right of all $a(f)^{*}$. In this form we have for all $\xi, \eta \in \mathscr{N}$

$$
\langle\xi, X \eta\rangle=\omega(X)\langle\xi, \eta\rangle,
$$

where $\omega(X)$ dentoes the constant term in the standard form. For each $\xi \in \mathscr{N} \backslash\{0\}$ we obtain a cyclic representation of $\mathscr{E} q(\mathscr{H})$, which is the GNS representation associated with the Fock state $\omega$. This is called the Fock representation. Since the cyclic subspaces generated from orthogonal Fock vectors are orthogonal, we can choose an orthonormal basis in $\mathcal{N}$, and thus obtain a direct sum of isomorphic copies of the Fock representation. The subspace on which this direct sum lives can be represented naturally as $\mathscr{K}_{\omega} \otimes \mathscr{N}$, where $\mathscr{K}_{\omega}$ denotes the GNS-Hilbert space of the Fock representation, and $a(f)\left\lceil\mathscr{K}_{\omega} \otimes \mathscr{N} \cong a_{\omega}(f) \otimes \mathbb{1}\right.$. The orthogonal complement of this invariant subspace is characterized by the property that it contains 
no non-zero Fock vectors. Note that the argument so far does not imply that there is any representation with $\mathscr{N} \neq\{0\}$. This will be the case if and only if the constant term in the standard form is indeed a positive functional on $\mathbf{E}^{q}(\mathscr{H})$. Equivalently, one has to check that the unique sesquilinear form on the vector space spanned by vectors of the form $a\left(f_{1}\right)^{*} \cdots a\left(f_{n}\right)^{*} \Omega$ which is computed by using the $q$-relations together with the equation $a(f) \Omega \equiv 0$, and the normalization condition $\|\Omega\|=1$, is positive definite. Bozejko and Speicher [BS] have shown this by reducing it to the positive definiteness of the function $\pi \mapsto q^{i(\pi)}$ on the permutation group of $n$ elements, where $i(\pi)=\mid\{(i, j) \mid i<j$ and $\pi(i)>\pi(j)\} \mid$ denotes the number of inversions in $\pi$.

EXAMPLE 2 (The case of a single relation). This case has been studied by many authors. Possibly the first occurrence is in the paper of Woronowicz [Wor], who considers the relations $\alpha \alpha^{*}+\nu^{2} \gamma \gamma^{*}=\mathbb{1}$, $\alpha^{*} \alpha+\gamma^{*} \gamma=\mathbb{1}, \alpha \gamma=\nu \gamma \alpha, \alpha \gamma^{*}=\nu \gamma^{*} \alpha$, and $\gamma^{*} \gamma=\gamma \gamma^{*}$. Thus by eliminating $\gamma$, one obtains $\alpha \alpha^{*}=\left(1-\nu^{2}\right) \mathbb{1}+\nu^{2} \alpha^{*} \alpha$, and $a=\left(1-\nu^{2}\right)^{-1 / 2} \alpha$ satisfies the $q$-relations with $q=\nu^{2}$ in the normalization used in this paper. Proposition 2 for this special case appears in Appendix 2 of [Wor]. We remark that multiplying the generators in the $q$-relations by a factor $\left(1-\nu^{2}\right)^{1 / 2}$ as suggested by [Wor] has the additional virtues of making the case $q=1$ less singular, and making the relations for

$$
|q|>1
$$

equivalent to those for $|q|<1$ with $a_{i}$ and $a_{i}^{*}$ interchanged. The algebras $\mathscr{E}^{q}(1)$ for a single relation then form a continuous field of $C^{*}$-algebras over the one-point compactification of the real line [Nic].

For later reference we include here a self-contained discussion of this simple case. By Proposition 1 a representation of the relation $a a^{*}-q a^{*} a=\mathbb{1}$ containing no Fock vectors has the property that $a=$ $(1-q)^{-1 / 2} u$, with a unitary $u$. Equivalently, in such representations we have the equation $a a^{*}=a^{*} a$. By Example 1 every representation is a direct sum of such an abelian representation and a multiple of the Fock representation. We can obtain an explicit picture of the Fock representation by taking the polar decomposition $a=v\left(a^{*} a\right)^{1 / 2}$. Since, by Proposition $1, a a^{*}$ is boundedly invertible it follows that $v^{*}=a^{*}\left(a a^{*}\right)^{-1 / 2}$ is an isometry, which lies in the $C^{*}$-algebra $\mathscr{E} q(\mathbb{C})$. Similarly, the projection $\mathbb{1}-v^{*} v=\operatorname{ker}\left(a^{*} a\right)$ onto the set $\mathscr{N}$ of Fock vectors lies in $\mathscr{E} q$. For studying the Fock representation we may thus take this projection to be one-dimensional in the representation space. 
Denoting by $|0\rangle$ the Fock vector, and $|n\rangle=\left(v^{*}\right)^{n}|0\rangle$ we get $v \rho^{2} v^{*}=$ $\mathbb{1}+q \rho^{2}$, where $\rho^{2}=a^{*} a$. Thus $v^{*}$ is a one-sided shift implementing the iteration on the spectrum used in the proof of Proposition 1, and we get $\left\langle n\left|\rho^{2}\right| m\right\rangle=\delta_{n m}\left(1-q^{n}\right) /(1-q)$. In particular, $\rho^{2}$ is a compact perturbation of $(1-q)^{-1} \mathbb{1}$. The algebra generated by $v$ thus contains $\rho^{2}$, and hence $\mathscr{E} q(\mathbb{C})$ is generated by the single isometry $v^{*}$. The ideal generated by $\mathbb{1}-v^{*} v$ is isomorphic to the compact operators, and the quotient by this ideal gives back the abelian representations. Thus all irreducible representations of $\mathscr{E} q(\mathbb{C})$ are quotients of the Fock representation, which therefore is faithful. Moreover, the algebras $\mathscr{E} q(\mathbb{C})$ are isomorphic for all $q \in(-1,1)$ to the well-known $C^{*}$ algebra $\mathscr{E}^{0}(\mathbb{C})[\mathbf{C o 1}, \mathbf{C o 2}]$ generated by a single isometry.

The following proposition shows that in a representation with several generators each single generator occurs in the Fock representation.

Proposition 4. For $\operatorname{dim} \mathscr{H} \geq 2$, any bounded representation of the $q$-relations, and every $0 \neq f \in \mathscr{H}, 0$ is in the spectrum of $a(f)^{*} a(f)$.

Proof. Since $a(f)$ generates a homomorphic image of $\mathscr{E} q(\mathbb{C})$ we only have to show that this homomorphism is faithful, i.e. that $u=$ $\|f\|^{-1} \sqrt{1-q} a(f)$ is not unitary. Suppose it were. Then from the commutation relations we would get for $g \perp f: u a(g)^{*} u=q a(g)^{*}$. Hence $\|a(g)\|=|q|\|a(g)\|$; because $|q|<1$ this implies $a(g)=0$, which implies $g=0$. This implies that $\mathscr{H}$ is one-dimensional.

The following is a typical feature of infinite $C^{*}$-algebras [Cun], i.e. algebras containing elements with $x^{*} x=\mathbb{1}$, but $x x^{*} \neq \mathbb{1}$, of which the Cuntz algebra is a prototype.

Corollary 5. For $-1<q<1$ and $\operatorname{dim} \mathscr{H} \geq 2, \mathscr{E} q(\mathscr{H})$ has no tracial state.

Proof. Suppose $\tau$ is a tracial state. Then its restriction to the subalgebra generated by $a(f)$ is also tracial. Since there is no finite trace on the compact operators, $\tau$ thus annihilates the corresponding ideal $\mathscr{I} \subset \mathscr{E} q(\mathbb{C})$. Hence $\tau\left(x^{*} z y\right)=\tau\left(y x^{*} z\right)=0$ for all $x, y \in \mathscr{E}^{q}(\mathscr{H})$ and $z \in \mathscr{I}$, which means that $\pi_{\tau}(z)=0$ for the cyclic representation associated with $\tau$. This contradicts Proposition 4.

Our main result regarding the $q$-relations is the stability of the algebra $\mathscr{E}^{0}$ under small $q$-deformations stated in the following proposition. It wil be proven in subsection 4.1 as a corollary of our stability 
result for the Cuntz relations (Theorem 9) with respect to a much larger class of perturbations.

Proposition 6. For $|q|<\sqrt{2}-1$ and all $d<\infty$, each $\mathscr{E}^{q}\left(\mathbb{C}^{d}\right)$ is $C^{*}$-isomorphic to $\mathscr{E}^{0}\left(\mathbb{C}^{d}\right)$. The isomorphism identifies $v_{i} \rho(q) \in$ $\mathscr{E}^{0}\left(\mathbb{C}^{d}\right)$ with $a_{i} \in \mathscr{E}^{q}\left(\mathbb{C}^{d}\right)$, where the $v_{i}$ are the generators in $\mathscr{E}^{0}\left(\mathbb{C}^{d}\right)$, and the function $q \mapsto \rho(q) \in \mathscr{E}^{0}\left(\mathbb{C}^{d}\right)$ is uniquely determined by $\rho(q) \geq$ 0 , and $\left(\mathbb{1}-\sum_{i} v_{i}^{*} v_{i}\right) \rho(q)=0$, and the condition that the $v_{i} \rho(q)$ satisfy the q-relations.

3. Stability of the Cuntz-Toeplitz algebra. In this section we will demonstrate a remarkable stability of the algebra $\mathscr{E}^{0}$ under practically all sufficiently small perturbations. The " $\Gamma$-relations" we consider will be of the form

$$
a_{i} a_{j}^{*}=\Gamma_{i j}\left(a_{1}, \ldots, a_{d}\right), \quad i, j=1, \ldots, d,
$$

where $\Gamma_{i j}$ are now functions (generally non-linear) of $a \equiv\left(a_{1}, \ldots, a_{d}\right)$ in the noncommutative $C^{*}$-functional calculus. Before elaborating on the definition of this functional calculus let us mention a few examples. The Cuntz relations are given by $\Gamma_{i j}=\delta_{i j} \mathbb{1}$. For the $q$-relations we set $\Gamma_{i j}(a)=\delta_{i j} \mathbb{1}+q a_{j}^{*} a_{i}$. In some detail we will later consider the relations $\Gamma_{i j}(a)=\delta_{i j} \mathbb{1}+q a_{i}^{*} a_{j}$. Further examples are quadratic polynomials whose constant term is an invertible matrix, and whose linear and second order terms are sufficiently small. We emphasize that the functions $\Gamma_{i j}$ need not be polynomials, and may contain fairly arbitrary continuous functions of their arguments. The exact conditions will be specified below. A fundamental common feature of all relations which we can treat is that the matrix $a_{i} a_{j}^{*}$ is invertible in the algebra $\mathscr{M}_{d}(\mathscr{A})$ of $d \times d$-matrices over the algebra $\mathscr{A} \ni a_{i}$. This property may seem surprising since $a_{i} a_{j}^{*}$ looks like the expression for a rank one matrix. In fact, $a_{i} a_{j}^{*}$ cannot be invertible for $a_{i}$ in any algebra with finite faithful trace, which underlines the infinite character of the $\Gamma$-relations.

By a function $f$ in the $C^{*}$-functional calculus [RW, SW] we mean a collection of functions $f_{\mathscr{A}}: \mathscr{A}^{d} \rightarrow \mathscr{A}$, one for each $C^{*}$-algebra $\mathscr{A}$, such that for any *-homomorphism $\pi: \mathscr{A} \rightarrow \mathscr{B}$ and any tuple $a_{1}, \ldots, a_{d}$ of arguments in $\mathscr{A}$ we have

$$
\pi f_{\mathscr{A}}\left(a_{1}, \ldots, a_{d}\right)=f_{\mathscr{B}}\left(\pi a_{1}, \ldots, \pi a_{d}\right) .
$$

Taking for $\pi$ the canonical injection from the $C^{*}\left(a_{1}, \ldots, a_{d}\right)$ into $\mathscr{A}$, we find that $f$ can always be evaluated in the algebra generated by 
its arguments. Since $f$ is also natural with respect to isomorphisms it suffices to define $f_{\mathscr{B}(\mathscr{K})}$ for some Hilbert space $\mathscr{K}$ in which any algebra generated by $d$ arguments has a faithful representation. Equation (7) only has to be postulated for all subalgebras of $\mathscr{B}(\mathscr{K})$, and then follows for arbitrary algebras. All polynomials in $d$ non-commuting variables and their adjoints are functions of the functional calculus. Conversely, if one restricts each argument by a uniform norm bound, any function of the calculus can be approximated uniformly by polynomials [RW].

In the sequel we will drop the subscript $\mathscr{A}$ for such functions. Tuples of operators satisfying the Cuntz relations $v_{i} v_{j}^{*}=\delta_{i j} \mathbb{1}$ will usually be denoted by $v_{i}, i=1, \ldots, d$. We show first how such operators emerge from representions of the $\Gamma$-relations with invertible $\Gamma$. For $a, b \in \mathscr{A}^{d}$ we define $\mathbf{e}(a, b) \in \mathscr{M}_{d}(\mathscr{A})$ by

$$
\mathbf{e}(a, b)_{i j}=a_{i} b_{j}^{*}
$$

LEMMA 7. Let $a_{1}, \ldots, a_{d} \in \mathscr{A}$. Suppose that $\mathbf{e}(a, a)$ is boundedly invertible. Then there are elements $v_{1}, \ldots, v_{d} \in \mathscr{A}$ such that with $\rho=\left(\sum_{i} a_{i}^{*} a_{i}\right)^{1 / 2}$ :

$$
v_{i} v_{j}^{*}=\delta_{i j} \mathbb{1}, \quad i, j=1, \ldots, d,
$$

$$
a_{i}=v_{i} \rho, \quad \rho \geq 0, \quad \text { and } \quad \rho=\left(\sum_{i} v_{i}^{*} v_{i}\right) \rho \text {. }
$$

Proof. Let $\mathscr{A}$ be faithfully represented on a Hilbert space $\mathscr{K}$, and let $\hat{a}: \mathscr{K} \rightarrow \mathscr{K}^{d}$ denote the operator $(\hat{a} \xi)_{i}=a_{i} \xi$, where the index $i$ refers to the $i$ th component in $\mathscr{K}^{d}$. Then $\mathbf{e}(a, a)=\hat{a} \hat{a}^{*}$. On the other hand, $\hat{a}^{*} \hat{a}=\sum_{i} a_{i}^{*} a_{i}=\rho^{2}$. Consider the polar decomposition of $\hat{a}$, i.e.

$$
\hat{a}=V\left(\hat{a}^{*} \hat{a}\right)^{1 / 2}=\left(\hat{a} \hat{a}^{*}\right)^{1 / 2} V,
$$

where $V: \mathscr{K} \rightarrow \mathscr{K}^{d}$ is a partial isometry, which we can write as $(V \xi)_{i}=v_{i} \xi$. In components the first equation becomes $a_{i}=v_{i} \rho$. Since $\left(\hat{a} \hat{a}^{*}\right)^{-1} \in \mathscr{M}_{d}(\mathscr{A})$ we have $V=\left(\hat{a} \hat{a}^{*}\right)^{-1 / 2} \hat{a}$, so that $v_{i} \in \mathscr{A}$. Furthermore, $V V^{*}=\mathbb{1}_{\mathscr{M}_{d}(\mathscr{A})}$, which means that $v_{i} v_{j}^{*}=\delta_{i j} \mathbb{1}$. On the other hand, $V^{*} V=\sum_{i} v_{i}^{*} v_{i}$ is the support projection of $\rho^{2}$, which proves the last equation.

Suppose now that some $\Gamma$-relations guarantee the invertibility of $\mathbf{e}(a, a)$. Then we know that the $C^{*}$-algebra generated by the $a_{i}$ is the same as the $C^{*}$-algebra generated by the $v_{i}$ and one additional 
element $\rho$. If we can show that $\rho$ is also in the algebra generated by the $v_{i}$, the algebras generated by the $v_{i}$ and by the $a_{i}$, respectively, are the same. Our strategy therefore is to fix the operators $v_{i}$ and to turn the $\Gamma$-relations into an equation for $\rho$. Substituting $a_{i}=v_{i} \rho$ (in short $a=v \rho$ ) in the $\Gamma$-relations we obtain the condition $v_{i} \rho^{2} v_{j}^{*}=$ $\Gamma_{i j}(v \rho)$. We multiply this with $v_{i}^{*}$ from the left and with $v_{j}$ from the right, sum over $i, j$, and use the support condition for $\rho$ to obtain

$$
\rho^{2}=\sum_{i, j=1}^{d} v_{i}^{*} \Gamma_{i j}(v \rho) v_{j}
$$

This equation is equivalent to the $\Gamma$-relations for $a=v \rho$, since we can restore the original form by multiplying this with $v_{i}$ and $v_{j}^{*}$, and using the Cuntz relations for the $v_{i}$. We will later solve equation (10) by contractive iteration. Since this involves taking the square root of $\rho$ we need the following Lipschitz estimate for square roots.

LEMMA 8. Let $\mathscr{A}$ be a $C^{*}$-algebra with identity, $\varepsilon>0$, and $x, y \geq$ $\varepsilon 1$. Then

$$
\|\sqrt{x}-\sqrt{y}\| \leq \frac{1}{2 \sqrt{\varepsilon}}\|x-y\| .
$$

Proof. By multiplying $x$ and $y$ with the same factor we may assume that $x=\mathbb{1}+\hat{x}, y=\mathbb{1}+\hat{y}$ with $\|\hat{x}\|,\|\hat{y}\| \leq \hat{\varepsilon}=1-\varepsilon$. Then

$$
\begin{aligned}
\|\sqrt{1+\hat{x}}-\sqrt{1+\hat{y}}\| & \leq \sum_{n=0}^{\infty}\left|\left(\begin{array}{c}
1 / 2 \\
n
\end{array}\right)\right|\left\|\hat{x}^{n}-\hat{y}^{n}\right\| \\
& \leq\|\hat{x}-\hat{y}\| \sum_{n=0}^{\infty}\left|\left(\begin{array}{c}
1 / 2 \\
n
\end{array}\right)\right| n \hat{\varepsilon}^{n-1} \\
& =\frac{1}{2}\|x-y\| \sum_{n=1}^{\infty}\left(\begin{array}{c}
-1 / 2 \\
n-1
\end{array}\right)(-\hat{\varepsilon})^{n-1} \\
& =\frac{1}{2}(1-\hat{\varepsilon})^{-1 / 2}\|x-y\| .
\end{aligned}
$$

For stating our main theorem it is convenient to introduce the norm

$$
\|a\|^{2}=\left\|\sum_{i=1}^{d} a_{i}^{*} a_{i}\right\|
$$

on $d$-tuples $a=\left(a_{1}, \ldots, a_{d}\right) \in \mathscr{A}^{d}$. Note that by the proof of Proposition 7 we have $\|\mathbf{e}(a, a)\|=\left\|\hat{a} \hat{a}^{*}\right\|=\left\|\hat{a}^{*} \hat{a}\right\|=\|a\|^{2}$. 
Theorem 9. Let $\Gamma_{i j}, i, j=1, \ldots, d$, be in the $C^{*}$-functional calculus of $d$ variables such that $\Gamma(a) \in \mathscr{M}_{d}(\mathscr{A})$ is hermitian for all $C^{*}$-algebras $\mathscr{A}$ and all $a \in \mathscr{A}^{d}$. Suppose there are constants $\mu, \varepsilon>$ $0, \lambda<2 \sqrt{\varepsilon}$ such that (with the norm given by (11))

(1) the $\Gamma$-relations $a_{i} a_{j}^{*}=\Gamma_{i j}(a)$ imply $\|a\|^{2} \leq \mu$.

(2) For arbitrary $a, b \in \mathscr{A}^{d}$ with $\|a\|^{2},\|b\|^{2} \leq \mu$ we have the estimates

$$
\|\Gamma(a)\| \leq \mu
$$

$$
\Gamma(a) \geq \varepsilon \mathbb{1}
$$

$$
\|\Gamma(a)-\Gamma(b)\| \leq \lambda\|a-b\| .
$$

Then there is a unique $\rho \in \mathscr{E}^{0}(d)$ such that $\rho \geq 0, \rho \sum_{i} v_{i}^{*} v_{i}=\rho$, and that $a_{i}=v_{i} \rho$ satisfies the $\Gamma$-relations, where the $v_{i}$ are the generators in $\mathscr{E}^{0}(d)$.

Moreover, this solution has the following universal property: if $\tilde{a}_{1}$, $\ldots, \tilde{a}_{d} \in \mathscr{A}$ satisfies the $\Gamma$-relations in any $C^{*}$-algebra $\mathscr{A}$, there is a unique $*$-homomorphism $\tilde{\pi}: \mathscr{E}^{0}(d) \rightarrow \mathscr{A}$ such that $\tilde{\pi}\left(v_{i} \rho\right)=\tilde{a}_{i}$ for all $i$.

Proof. Let $\mathscr{A}$ be any $C^{*}$-algebra containing elements $\tilde{v}_{i}, i=$ $1, \ldots, d$, satisfying the Cuntz relations. We will iterate

$$
f(x)=\left(\sum_{i j} \tilde{v}_{i}^{*} \Gamma_{i j}(\tilde{v} x) \tilde{v}_{j}\right)^{1 / 2}
$$

on the set

$$
X=\left\{x \in \mathscr{A} \mid 0 \leq x, x^{2} \leq \mu p\right\},
$$

where $(\tilde{v} x)_{i}=\tilde{v}_{i} x$, and $p=\sum_{i} \tilde{v}_{i}^{*} \tilde{v}_{i}$. Then by equation (10) the fixed points of $f$ are precisely those $x \in \mathscr{A}$ with $x \geq 0$ and $x p=x$ such that $\tilde{v} x$ satisfies the $\Gamma$-relations. The set $X$ is invariant, because $\|\tilde{v} x\|^{2} \leq\|x\|^{2}$, so $x^{2} \leq \mu p$ implies $\left\|f(x)^{2}\right\| \leq\|\Gamma(\tilde{v} x)\| \leq \mu$ by (a), and $f(x)^{2}=p f(x)^{2} p \leq \mu p$ by the Cuntz relations. By assumption (b) $\Gamma(\tilde{v} x) \geq \varepsilon \mathbb{1}$ for $x \in X$; hence $f(x)^{2} \geq \varepsilon p$. The square root is thus well defined, and by Lemma 8 (applied in the algebra $p \mathscr{A} p$ with unit $p$ ) we get from (c) the estimate

$$
\begin{aligned}
\|f(x)-f(y)\| & \leq \frac{1}{2 \sqrt{\varepsilon}}\left\|f(x)^{2}-f(y)^{2}\right\| \\
& \leq \frac{\lambda}{2 \sqrt{\varepsilon}}\|\tilde{v} x-\tilde{v} y\| \leq \frac{\lambda}{2 \sqrt{\varepsilon}}\|x-y\| .
\end{aligned}
$$


Thus $f$ is contractive, and has a unique fixed point $\tilde{\rho}$ by the Banach Contraction Theorem. We apply this firstly in the case $\mathscr{A}=\mathscr{E}^{0}(d)$ with $\tilde{v}=v$ to obtain $\rho \in \mathscr{E}^{0}(d)$ as claimed in the theorem.

Now let $\tilde{a} \in \mathscr{A}^{d}$ satisfy the $\Gamma$-relations. Then by assumption (1) of the theorem, $\|\tilde{a}\|^{2} \leq \mu$, which implies that $\mathbf{e}(\tilde{a}, \tilde{a})=\Gamma(\tilde{a}) \geq \varepsilon \mathbb{1}$ is invertible. Hence, by Lemma $7, \tilde{a}=\tilde{v} \tilde{\rho}$, and $\tilde{\rho}$ must be the unique fixed point of the iteration in $\mathscr{A}$. On the other hand, by the universal property of $\mathscr{E}^{0}(d)$ for the Cuntz relations there is a unique homomorphism $\tilde{\pi}: \mathscr{E}^{0}(d) \rightarrow \mathscr{A}$ such that $\tilde{\pi}\left(v_{i}\right)=\tilde{v}_{i}$. By the homomorphism property (7) of the functional calculus we have

$$
\begin{aligned}
\Gamma_{i j}\left(\tilde{v}_{1} \tilde{\pi}(\rho), \ldots, \tilde{v}_{d} \tilde{\pi}(\rho)\right) & =\Gamma_{i j}\left(\tilde{\pi}\left(v_{1} \rho\right), \ldots, \tilde{\pi}\left(v_{d} \rho\right)\right) \\
& =\tilde{\pi}\left(\Gamma_{i j}\left(v_{1} \rho, \ldots, v_{d} \rho\right)\right) \\
& =\tilde{\pi}\left(v_{i} \rho^{2} v_{j}^{*}\right)=\tilde{v}_{i} \tilde{\pi}(\rho)^{2} \tilde{v}_{j}^{*} .
\end{aligned}
$$

Hence $\tilde{\pi}(\rho)$ is also a fixed point of the iteration in $\mathscr{A}$, and we must have $\tilde{\pi}(\rho)=\tilde{\rho}$, and $\tilde{\pi}\left(a_{i}\right)=\tilde{v}_{i} \tilde{\rho}=\tilde{a}_{i}$.

\section{Applications.}

4.1. The q-relations. In equation (8) we have introduced the dyads $\mathbf{e}(a, b)$ for $a, b \in \mathscr{A}^{d}$. Here we need a second kind of dyad, which is equal to $\mathbf{e}(\cdot, \cdot)$ when $a_{i}$ and $b_{i}$ commute. We set

$$
\mathbf{f}(a, b)_{i j}=b_{j}^{*} a_{i}, \quad \mathbf{e}(a, b)_{i j}=a_{i} b_{j}^{*} .
$$

Using these dyads we can write the $q$-relations in the compact form

$$
\mathbf{e}(a, a)=\mathbb{1}+q \mathbf{f}(a, a) .
$$

The norm bounds needed for the application of Theorem 9 are given in the next lemma.

Lemma 10. Let $a, b \in \mathscr{A}^{d}$. Then $\|\mathbf{e}(a, a)\|=\left\|\sum a_{i}^{*} a_{i}\right\| \equiv\|a\|^{2}$, and

$$
\|\mathbf{f}(a, b)\| \leq\|a\|\|b\|, \quad\|\mathbf{e}(a, b)\| \leq\|a\|\|b\| .
$$

Moreover, $\left\|a^{*}\right\|^{2} \leq d\|a\|^{2}$.

Proof. The equation for $\|\mathbf{e}(a, a)\|$ was already noted before Theorem 9.

$$
\left(\mathbf{e}(a, b)^{*} \mathbf{e}(a, b)\right)_{i j}=(\mathbf{e}(b, a) \mathbf{e}(a, b))_{i j}=b_{i}\left(\sum_{k} a_{k}^{*} a_{k}\right) b_{j}^{*} .
$$


The last expression can be written as $\hat{b}\left(\sum_{k} a_{k}^{*} a_{k}\right) \hat{b}^{*}$ with a $d \times 1$ matrix $\hat{b}$. Estimating the bracket by its norm we get $\mathbf{e}(a, b)^{*} \mathbf{e}(a, b) \leq$ $\|a\|^{2} \mathbf{e}(b, b)$, and $\|\mathbf{e}(a, b)\|^{2} \leq\|a\|^{2}\|\mathbf{e}(b, b)\|$.

Similarly, we get

$$
\left(\mathbf{f}(a, b)^{*} \mathbf{f}(a, b)\right)_{i j}=(\mathbf{f}(b, a) \mathbf{f}(a, b))_{i j}=\sum_{k} a_{k}^{*} b_{i} b_{j}^{*} a_{k}=\Phi\left(b_{i} b_{j}^{*}\right)
$$

with the completely positive map $\Phi(x)=\sum_{k} a_{k}^{*} x a_{k}$. Hence

$$
\mathbf{f}(a, b)^{*} \mathbf{f}(a, b) \leq\|\mathbf{e}(b, b)\| \Phi(\mathbb{1}), \quad \text { and } \quad\|\mathbf{f}(a, b)\|^{2} \leq\|b\|^{2}\|a\|^{2} .
$$

Finally we get $\left\|a^{*}\right\|=\left\|\sum a_{k} a_{k}^{*}\right\| \leq \sum_{k=1}^{d}\left\|a_{k}\right\|^{2} \leq d\left\|\sum a_{k}^{*} a_{k}\right\|$, as claimed.

Proof of Proposition 6. With $\Gamma(a)=\mathbb{1}+q \mathbf{f}(a, a)$ we get

$$
\|\Gamma(a)\| \leq 1+|q|\|\mathbf{f}(a, a)\| \leq 1+|q|\|a\|^{2} .
$$

Hence the $q$-relations imply $\|a\|^{2} \leq 1+|q|\|a\|^{2}$, and $\|a\|^{2} \leq$ $(1-|q|)^{-1} \equiv \mu$. For $\|a\|^{2} \leq \mu$ we get $\|\Gamma(a)\| \leq \mu$ as required in condition (2a) of Theorem 9. Furthermore,

$$
\Gamma(a) \geq \mathbb{1}(1-|q|\|\mathbf{f}(a, a)\|) \geq \mathbb{1}(1-|q| \mu)=\varepsilon \mathbb{1}
$$

with $\varepsilon=(1-2|q|) /(1-|q|)$. Hence for $\Gamma(a)$ to be bounded away from zero as required in condition $(2 b)$ we need $|q|<1 / 2$. Using the bilinearity of $\mathbf{f}(a, b)$ we get the Lipschitz bound

$$
\begin{aligned}
\|\Gamma(a)-\Gamma(b)\| & =|q|\|\mathbf{f}(a, a)-\mathbf{f}(b, b)\| \\
& \leq|q|\|a-b\|(\|a\|+\|b\|) \leq 2|q| \sqrt{\mu}\|a-b\| .
\end{aligned}
$$

With $\lambda=2|q| \sqrt{\mu}$ the contractivity condition then becomes $|q|<$ $\sqrt{1-2|q|}$, or $|q|<\sqrt{2}-1$.

For a possible extension of Proposition 6 to a larger interval, perhaps even to the whole range $q \in(-1,1)$, it is crucial to improve the bounds

$$
\frac{1-2|q|}{1-|q|} \mathbb{1} \leq \mathbf{e}(a, a) \leq \frac{1}{1-|q|} \mathbb{1}
$$

for $a$ satisfying the $q$-relations, which were obtained in the above proof. The upper bound is fairly good. In fact, for $q \geq 0$ the upper bound is an equality since by Proposition 1 each diagonal element in $\mathbf{e}(a, a)$ has norm $(1-q)^{-1}$. As a corollary of this observation we find that in every representation with $d+1$ generators there 
are many vectors on which $\sum_{i}^{d} a_{i}^{*} a_{i}$ is small (namely those on which $a_{d+1}^{*} a_{d+1}$ nearly attains its maximum). The existence of such "almost Fock" vectors for a subset of generators can be seen as a generalization of Proposition 4, and can be extended also to some negative values of $q$. For $q<0$ the upper bound in (14) is the best possible $d$-independent bound. (The vectors in Fock space arising from (anti-) symmetrization of $a_{1}^{*} \cdots a_{n}^{*}|0\rangle$ are eigenvectors of $\sum_{i=1}^{d} a_{i}^{*} a_{i}$ with eigenvalue $\left(1-( \pm q)^{n}\right) /(1 \mp q)$.) However, for given finite $d$ the bound can be improved. For example, for $d=2$ we get

$$
\begin{aligned}
& \mathbf{e}(a, a)=\left(\begin{array}{cc}
a_{1} a_{1}^{*} & 0 \\
0 & a_{2} a_{2}^{*}
\end{array}\right)+q\left(\begin{array}{cc}
0 & a_{2}^{*} a_{1} \\
a_{1}^{*} a_{2} & 0
\end{array}\right), \\
&\left(\begin{array}{cc}
0 & a_{2}^{*} a_{1} \\
a_{1}^{*} a_{2} & 0
\end{array}\right)^{2}=\left(\begin{array}{cc}
a_{2}^{*} a_{1} a_{1}^{*} a_{2} & 0 \\
0 & a_{1}^{*} a_{2} a_{2}^{*} a_{1}
\end{array}\right) \leq\left(\begin{array}{cc}
a_{2}^{*} a_{2} & 0 \\
0 & a_{1}^{*} a_{1}
\end{array}\right) \leq \mathbb{1},
\end{aligned}
$$

where we have used the bound $a a^{*} \leq 1$ from Corollary 2. Hence

$$
\|a\|^{2}=\|\mathbf{e}(a, a)\| \leq 1+|q| \text { for } d=2 .
$$

We can insert this into the bound $\mathbf{e}(a, a) \geq(1-|q|\|\mathbf{f}(a, a)\|) \mathbb{1} \geq$ $\left(1-|q|\|a\|^{2}\right) \mathbb{1}$ to get an improvement of the lower bound in (14):

$$
\mathbf{e}(a, a) \geq 1-|q|-|q|^{2} \text { for } d=2 \text {. }
$$

Hence $\mathbf{e}(a, a)$ is boundedly invertible for $q>-\phi^{-1}=-\frac{1}{2}(\sqrt{5}-1)$. It is not clear what the best lower bounds on $\mathbf{e}(a, a)$ are. Numerical evidence from Fock space suggests that $\mathbf{e}(a, a)$ might be bounded away from zero for all $-1<q<1$ with bounds going to zero at the endpoints of the interval.

4.2. The relations $a_{i} a_{j}^{*}-q a_{i}^{*} a_{j}=\delta_{i j} \mathbb{1}$. These relations are maybe the most natural choice of a quadratic polynomial $\Gamma$ apart from the $q$-relations. The only difference between these is the position of the indices in the term containing the parameter $q$. In terms of the dyads introduced in $\S 4.1$ we can write these relations as

$$
\mathbf{e}(a, a)=\mathbb{1}+q \mathbf{e}\left(a^{*}, a^{*}\right) .
$$

The two sets of relations are identical if either $d=1$, or $q=0$. Thus we can directly take over Proposition 1 and Corollary 2. The existence of any bounded realizations for general $q$ is not immediately clear. But if for any values of $(q, d)$ there is any bounded realization, then there is a universal algebra $\mathscr{D}^{q}(d)$ satisfying Proposition 3 with one modification: The relations are now not invariant under the unitary 
group $U_{d}$, but only under the orthogonal subgroup $O_{d}$, and correspondingly we should take a real Hilbert space $\mathscr{H}$ as the analogue of the complex space $\mathscr{H}$ in Proposition 3. Even though multiplication of test functions with a phase thus makes no more sense, the automorphism " $\mathscr{D}^{q}(\eta \mathbb{1})$ " taking each $a(f)$ into $\eta a(f)$ is still well defined by the univeral property of $\mathscr{D}^{q}(d)$, and Proposition 3(4) also carries over.

Applying Theorem 9 to these relations yields

Proposition 11. For $d \in \mathbb{N}$, and $|q|<1 /(d \phi)=(\sqrt{5}-1) /(2 d)$ the functions $\Gamma_{i j}(a)=\delta_{i j} \mathbb{1}+a_{i}^{*} a_{j}$ satisfy the conditions of Theorem 9 . Consequently, $\mathscr{D}^{q}(d)$ exists for these values of $q$ and is isomorphic to $\mathscr{E}^{0}\left(\mathbb{C}^{d}\right)$.

Proof. Since $\mathbf{e}(a, a) \geq 0$ it pays in this case to distinguish $q>0$ and $q<0$. For $q>0$ we apply Lemma 10 to get $\|\Gamma(a)\| \leq 1+$ $q\left\|\mathbf{e}\left(a^{*}, a^{*}\right)\right\| \leq 1+q d\|a\|^{2}$, and set $\mu=(1-q d)^{-1}$ in Theorem 9 . Clearly, we can set $\varepsilon=1$. With $\lambda=2 q d \sqrt{\mu}$ the contractivity estimate is $q d<\sqrt{1-q d}$, or $q d<\phi^{-1}$.

For $q<0$ we can take $\mu=1$. Then

$$
\Gamma(a) \geq \mathbb{1}\left(1-|q|\left\|\mathbf{e}\left(a^{*}, a^{*}\right)\right\|\right) \geq \varepsilon \mathbb{1}
$$

with $\varepsilon=(1-|q| d)$. The Lipschitz bound is $\lambda=2|q| d$, so contractivity holds once more for $|q| d<\sqrt{1-|q| d}$.

The fact that the interval for $q$ for which our technique works now depends on $d$ came in through the estimate $\left\|a^{*}\right\|^{2} \leq d\|a\|^{2}$ in Lemma 10, which may appear to be exceedingly crude. However, this $d$-dependence is typical for the relations (17). We will show this now by using the idea of Proposition 1 to obtain rather detailed necessary conditions for such representations. The core of the idea is contained in the following proposition:

Proposition 12. Let $a_{i}, i=1, \ldots, d$, be bounded operators satisfying the rleations (17). Let $T=\sum_{i=1}^{d} a_{i}^{*} a_{i}$, and consider the function $f(x)=1+q d+q^{2} x$. Then

$$
x \in \sigma(T) \Leftrightarrow f(x) \in \sigma(T),
$$

provided that both $f(x) \neq 1$ and $f(x) \neq 0$.

Proof. We consider two $d \times 1$-matrices with entries in $\mathscr{A}$, namely $A_{i}=a_{i}$, and $\widehat{A_{i}}=a_{i}^{*}$. This somewhat redundant notation is necessary 
to keep apart the different meaning of adjoints. We can then write

$$
\begin{aligned}
& \mathbf{e}(a, a)=A A^{*}, \quad \mathbf{e}\left(a^{*}, a^{*}\right)=\widehat{A} \widehat{A}^{*}, \quad A A^{*}=\mathbb{1}+q \widehat{A} \widehat{A}^{*}, \\
& T:=\sum_{i} a_{i}^{*} a_{i}=A^{*} A, \quad \widehat{T}:=\sum_{i} a_{i} a_{i}^{*}=\widehat{A}^{*} \widehat{A}, \quad \widehat{T}=d \mathbb{1}+q T .
\end{aligned}
$$

Now suppose that $x$ satisfies the two conditions in the proposition. Then $x \in \sigma(T) \Leftrightarrow d+q x \in \sigma(\widehat{T})$. Since we have assumed $d+q x=$ $(1 / q)(f(x)-1) \neq 0$ this is in turn equivalent to $d+q x \in \sigma\left(\widehat{A}^{*} \widehat{A}\right)$, and $d+q x \in \sigma\left(\widehat{A} \widehat{A}^{*}\right)$. This is equivalent to $(1+q(d+q x))=f(x) \in$ $\sigma\left(A A^{*}\right)$. Since we have assumed that $f(x) \neq 0$ this is equivalent to $f(x) \in \sigma(T)$.

Proposition 13. Let $a_{1}, \ldots, a_{d}$ with $d>1$ be bounded operators satisfying the relations (17). Let $T=\sum_{i=1}^{d} a_{i}^{*} a_{i}$, and $x_{\infty}=$ $(1+q d) /\left(1-q^{2}\right)$. For $n \in \mathbb{N}, n \geq 0$ let $\lambda_{n}=x_{\infty}\left(1-q^{2 n}\right)$ and $\mu_{n}=x_{\infty}+\left(1-x_{\infty}\right) q^{2 n}$. Then the spectrum of $T$ is contained in

$$
\left\{\lambda_{n} \mid n \geq 0\right\} \cup\left\{\mu_{n} \mid n \geq 0\right\} \cup\left\{x_{\infty}\right\} .
$$

Moreover, one has

(1) For $q \geq-d^{-1}$, all $\mu_{n}$ are in the spectrum, and either all or no $\lambda_{n}$. Moreover, for $q \neq 0, \lambda_{n} \neq \mu_{m}$ for all $n, m \geq 0$.

(2) For $q<-d^{-1}$, the spectrum of $T$ is of the form

$$
\left\{\mu_{0}, \mu_{1}, \ldots, \mu_{N-1}\right\}
$$

with $\mu_{N}=0$ for some finite $N$. Thus representations can exist only for the discrete set of values $q$ for which the equation $\mu_{N}=0$, or equivalently

$$
q^{2 N}=\frac{1+q d}{q(d+q)}
$$

has a solution.

Proof. If $x \in \sigma(T), x \neq x_{\infty}$, the iteration of $f^{-1}$, with $f$ as in Proposition 12, yields an unbounded sequence. Thus, by Proposition 12 the iterates must either hit 0 or 1 , which means that either $x=$ $\lambda_{n}$ or $x=\mu_{n}$ for some $n \in \mathbb{N}$. Next observe that Proposition 1 and the argument in Proposition 4 show that $0 \in \sigma\left(a_{1}^{*} a_{1}\right)$. Thus by Proposition 1 we can choose a non-zero $\xi \in \operatorname{ker}\left(a_{1}^{*} a_{1}\right)=\operatorname{ker}\left(a_{1}\right)$. Now the vector $(\xi, 0, \ldots, 0)$ is in $\operatorname{ker}\left(\widehat{A} \widehat{A}^{*}\right)$. This implies $1 \in \sigma\left(A A^{*}\right)$, and $\mu_{0} \in \sigma(T)$.

If $q>-1 / d$ then $x_{\infty}$ is positive, and the sequence $\lambda_{n}$ is strictly increasing towards $x_{\infty}$. If in addition $q \leq 0$, then $0 \leq x_{\infty} \leq 1$, 
and the sequence $\mu_{n}$ is decreasing towards $x_{\infty}$, implying that the sequences are disjoint. If $q>0$, both sequences are increasing. They do not intersect, because $\mu_{n}>\mu_{0}=1>0=\lambda_{0}$, and $\lambda_{n} \geq \lambda_{1}=$ $1+q d>1=\mu_{0}$ for $n>0$, and the inverse iteration exists.

If $q<-1 / d$, then $x_{\infty}$ and all $\lambda_{n}, n>0$, are strictly negative. Hence $0 \notin \sigma(T) . \mu_{n}$ is strictly decreasing. Thus we can only have that $\mu_{N}=0$ for some $N \in \mathbb{N}$.

Acknowledgments. R. F. W. acknowledges stimulating conversations with Burkhard Kümmerer during an enjoyable stay in Tübingen. We thank Roland Speicher for providing us with his recent works on the topic. P. J. acknowledges discussions with G. L. Price toward the end of this project. R. F. W. was supported by a fellowship from the DFG (Bonn). We would like to thank the referee for his constructive and detailed criticism, and for emphasizing the connection of our work to [Wor].

\section{REFERENCES}

[Bie] L. C. Biedenharn, The quantum group $S U_{q}(2)$ and a q-analogue of the boson operators, J. Phys. A, 22 (1989), L873-L878.

[BS] M. Bozejko and R. Speicher, An example of a generalized Brownian motion, Commun. Math. Phys., 137 (1991), 519-531.

[BDF] L. G. Brown, R. G. Douglas and P. A. Fillmore, Extensions of $C^{*}$-algebras and K-homology, Ann. Math., 105 (1977), 265-324.

[Chr] E. Christensen, Close operator algebras, In: [HG].

[Co1] L. A. Coburn, The $C^{*}$-algebra generated by an isometry, I, Bull. Amer. Math. Soc., 73 (1967), 722-726.

[Co2] - The $C^{*}$-algebra generated by an isometry, II, Trans. Amer. Math. Soc., 137 (1969), 211-217.

[Con] J. B. Conway, Subnormal Operators, Pitman Books Ltd., London, 1981.

[Cun] J. Cuntz, Simple $C^{*}$-algebras generated by isometries, Commun. Math. Phys., 57 (1977), 173-185.

[Das] C. Daskaloyannis, Generalized deformed oscillator and nonlinear algebras, J. Phys. A., 24 (1991), L789-L794.

[DR] S. Doplicher and J. E. Roberts, Duals of compact Lie groups realized in the Cuntz algebras and their actions on $C^{*}$-algebras, J. Funct. Anal., 74 (1987), 96-120.

[Dou] R. G. Douglas, Banach Algebra Techniques in Operator Theory, Academic Press, New York, 1972.

[Eva] D. E. Evans, $O n \mathscr{O}_{n}$, Publ. R.I.M.S., Kyoto, 16 (1980), 915-925.

[Fiv] D. I. Fivel, Interpolation between Fermi and Bose statistics using generalized commutators, Phys. Rev. Lett., 65 (1990), 3361-3364.

[Ger] M. Gerstenhaber, The cohomology structure of an associative ring, Ann. Math., 78 (1963), 267-288.

[Gre] O. W. Greenberg, Particles with small violations of Fermi or Bose statistics, Phys. Rev. D., 43 (1991), 4111-4120. 
[HG] M. Hazewinkel and M. Gerstenhaber (eds.), Deformation Theory of Algebras and Structures and Applications, Kluwer Academic Publishers, Dordrecht, 1988.

[Mcf] A. J. Macfarlane, On q-analogues of the quantum harmonic oscillator and the quantum group $S U(2)_{q}$, J. Phys. A., 22 (1989), 4581-4588.

[Moh] R. N. Mohapatra, Infinite statistics and a possible small violation of the Pauli principle, Phys. Lett. B., 242 (1990), 407-411.

[vNe] J. von Neumann, Die Eindeutigkeit der Schrödingerschen Operatoren, Math. Ann., 104 (1931), 570-578.

[Nic] A. Nica, On a quantization related to the Toeplitz algebra, Preprint, Berkeley, 1992.

[OKK] K. Odaka, T. Kishi and S. Kamefuchi, On quantization of simple harmonicoscillators, J. Phys. A, 24 (1991), L591-L596.

[RW] G. A. Raggio and R. F. Werner, Quantum statistical mechanics of general mean field systems, Helvet. Phys. Acta., 62 (1989), 980-1003.

[Ros] A. L. Rosenberg, The unitary irreducible representations of the quantum Heisenberg algebra, Comm. Math. Phys., 144 (1992), 41-51.

[SW] L. M. Schmitt and R. F. Werner, Non-commutative functional calculus, in preparation.

[Spei] R. Speicher, A non-commutative central limit theorem, Math. Z., 209 (1992), 55-66.

[Wor] S. L. Woronowicz, Twisted $S U(2)$ group. An example of non-commutative differential calculus, Publ. R.I.M.S., Kyoto, 23 (1987), 117-181.

Received May 15, 1992 and in revised form September 10, 1992. The first author was suported in part by the NSF (USA) and NATO.

UNIVERSITY OF IOWA

IOWA CITY, IA 52242

E-mail address: jorgen@math.uiowa.edu

UNIVERSITÄT OSNABRÜCK

D-4500 OSNABRÜCK, GERMANY

Current address: University of Aizu

Aizu-Wakamatsu, Fukushima 965

Japan

AND

FB PHYSIK

UNIVERSITÄT OSNABRÜCK 49069 OSNABRÜCK, GERMANY

E-mail address: reinwer@dosuni.rz.uni-osnabrueck.de 



\section{PACIFIC JOURNAL OF MATHEMATICS}

Founded by

E. F. BECKENBACH (1906-1982) F. Wolf (1904-1989)

\section{EDITORS}

Sun-Yung A. Chang

(Managing Editor)

University of California

Los Angeles, CA 90024-1555

chang@math.ucla.edu

\section{F. Michael Christ}

University of California

Los Angeles, CA 90024-1555

christ@math.ucla.edu

Herbert Clemens

University of Utah

Salt Lake City, UT 84112

clemens@math.utah.edu

\author{
ThOMAs ENRIGHT \\ University of California, San Diego \\ La Jolla, CA 92093 \\ tenright@ucsd.edu \\ Nicholas ERcolaNI \\ University of Arizona \\ Tucson, AZ 85721 \\ ercolani@math.arizona.edu \\ R. FINN \\ Stanford University \\ Stanford, CA 94305 \\ finn@gauss.stanford.edu \\ VAUGHAN F. R. Jones \\ University of California \\ Berkeley, CA 94720 \\ vfr@math.berkeley.edu
}

\author{
STEVEn KerCKHOFF \\ Stanford University \\ Stanford, CA 94305 \\ spk@gauss.stanford.edu
}

Martin ScharlemanN

University of California

Santa Barbara, CA 93106

mgscharl@math.ucsb.edu

\author{
Harold Stark \\ University of California, San Diego \\ La Jolla, CA 92093 \\ V. S. VARADARAJAN \\ University of California \\ Los Angeles, CA 90024-1555 \\ vsv@math.ucla.edu
}

\section{SUPPORTING INSTITUTIONS}

UNIVERSITY OF ARIZONA

UNIVERSITY OF BRITISH COLUMBIA

CALIFORNIA INSTITUTE OF TECHNOLOGY

UNIVERSITY OF CALIFORNIA

UNIVERSITY OF MONTANA

UNIVERSITY OF NEVADA, RENO

NEW MEXICO STATE UNIVERSITY

OREGON STATE UNIVERSITY

\author{
UNIVERSITY OF OREGON \\ UNIVERSITY OF SOUTHERN CALIFORNIA \\ STANFORD UNIVERSITY \\ UNIVERSITY OF HAWAII \\ UNIVERSITY OF UTAH \\ WASHINGTON STATE UNIVERSITY \\ UNIVERSITY OF WASHINGTON
}

The Supporting Institutions listed above contribute to the cost of publication of this Journal, but they are not owners or publishers and have no responsibility for its content or policies.

Mathematical papers intended for publication in the Pacific Journal of Mathematics should be in typed form or offset-reproduced (not dittoed), double spaced with large margins. Please do not use built up fractions in the text of the manuscript. However, you may use them in the displayed equations. Underline Greek letters in red, German in green, and script in blue. The first paragraph must be capable of being used separately as a synopsis of the entire paper. In particular it should contain no bibliographic references. Please propose a heading for the odd numbered pages of less than 35 characters. Manuscripts, in triplicate, may be sent to any one of the editors. Please classify according to the 1991 Mathematics Subject Classification scheme which can be found in the December index volumes of Mathematical Reviews. Supply name and address of author to whom proofs should be sent. All other communications should be addressed to the managing editor, or Julie Honig, University of California, Los Angeles, California 90024-1555.

There are page-charges associated with articles appearing in the Pacific Journal of Mathematics. These charges are expected to be paid by the author's University, Government Agency or Company. If the author or authors do not have access to such Institutional support these charges are waived. Single authors will receive 75 free reprints; joint authors will receive a total of 100 free reprints. Additional copies may be obtained at cost in multiples of 50 .

The Pacific Journal of Mathematics (ISSN 0030-8730) is published monthly except for July and August. Regular subscription rate: $\$ 215.00$ a year (10 issues). Special rate: $\$ 108.00$ a year to individual members of supporting institutions.

Subscriptions, orders for numbers issued in the last three calendar years, and changes of address should be sent to Pacific Journal of Mathematics, P.O. Box 4163, Berkeley, CA 94704-0163, U.S.A. Old back numbers obtainable from Kraus Periodicals Co., Route 100, Millwood, NY 10546.

The Pacific Journal of Mathematics at University of California, c/o Department of Mathematics, 981 Evans Hall, Berkeley, CA 94720 (ISSN 0030-8730) is published monthly except for July and August. Second-class postage paid at Berkeley, CA 94704, and additional mailing offices. POSTMASTER: send address changes to Pacific Journal of Mathematics, P.O. Box 4163, Berkeley, CA 94704-0163.

PUBLISHED BY PACIFIC JOURNAL OF MATHEMATICS at University of California, Berkeley, CA 94720, A NON-PROFIT CORPORATION

This publication was typeset using $\mathcal{A} \mathcal{M} \mathcal{S}-\mathrm{T}_{\mathrm{E}} \mathrm{X}$,

the American Mathematical Society's $T_{E} X$ macro system.

Copyright (c) 1994 by Pacific Journal of Mathematics 


\section{PACIFIC JOURNAL OF MATHEMATICS}

Volume $165 \quad$ No. $1 \quad$ September 1994

The effect of dimension on certain geometric problems of irregularities of 1 distribution

RALPH ALEXANDER

The structure of $\mathrm{sl}(2,1)$-supersymmetry: irreducible representations and primitive ideals

Didier ARnAL, HÉdi BENAMOR and GEORges PinCZON

Periods and Lefschetz zeta functions

Josefina CASASAYas, JaUme Llibre and ANA Nunes

On closed hypersurfaces of constant scalar curvatures and mean curvatures in 67 $S^{n+1}$

\section{SHAOPING CHANG}

$R$-groups and elliptic representations for $\mathrm{SL}_{n}$

DAVID GOLDBERG

The boundary distortion of a quasiconformal mapping

JuHA HeINONEN and PEKKA KosKela

Strongly approximately transitive group actions, the Choquet-Deny theorem, 115 and polynomial growth

\section{WOJCIECH JAWORSKI}

$q$-canonical commutation relations and stability of the Cuntz algebra

PALle E. T. Jorgensen, L. M. Schmitt and Reinhard Frank

WERNER

Complete open manifolds of non-negative radial curvature

YOSHIROH MACHIGASHIRA

Perturbations of certain reflexive algebras

DAVID RYDER PITTS

Interpolation submanifolds of the unitary group 